\title{
Trabajo social y valoración gerontogeriátrica: tamizaje social en el Centro Gerontológico Guillermina Loor de Moreno
}

Social work and gerontogeriatric assessment: social screening in the Guillermina Loor de Moreno Gerontological Center

Juan José Giler Basurto 1

Eva Margarita Alcívar Medranda²

RECIBIDO: 30 DE ABRIL DE 2020

ACEPTADO: 5 DE JUNIO DE 2020

\section{RESUMEN}

El artículo planteó como objetivo determinar los procesos de intervención del equipo interdisciplinario en la valoración gerontogeriatrica de los residentes del Centro Gerontológico Guillermina Loor de Moreno. El trabajo consta de dos apartados interrelacionados entre sí: el primero describe la función que tiene el Trabajador Social en el Centro Gerontológico (Trabajo Social Gerontológico) como líder del

\footnotetext{
' Egresado de Trabajo Social, Universidad Técnica de Manabí, Ecuador; gilerbasurtojuanjose@gmail.com, ORCID: https://orcid.org/0000-0002-4707-5232

Google Scholar: https://scholar.google.es/citations?hl=es\&user=|BxWxukAAAAJ

2 Magister en Educación y Desarrollo Social, Licenciada en Trabajo Social, Docente Investigadora, Universidad Técnica de Manabí, Ecuador; evaportoviejol@gmail.com, ORCID: https://orcid.org/00000003-1839-2535

Google Scholar: https://scholar.google.es/citations?hl=es\&user=6M4WSm8AAAAJ
}

Socialium revista científica de Ciencias Sociales, Vol 4 - No. 2, julio - diciembre 2020, pág. 97-110.

DOI https://doi.org/10.26490/uncp.sl.2020.4.2.571 


\section{8}

equipo interdisciplinario, el segundo se centra en la valoración gerontogeriatrica que se obtiene a través del Plan de Intervención Individual Integral (PIII) que realizan los profesionales del equipo, quien actúa sujeto a la normativa técnica del Ministerio de Inclusión Económica y Social (MIES). Esto permitió entender y procesar mejor los protocolos de intervención en el campo del adulto mayor. Los métodos descriptivos - exploratorio, bibliográfico e inductivo-deductivo permitieron sintetizar la información de cada proceso, la técnica de la entrevista facilitó adquirir información relevante en la atención que reciben éste grupo vulnerable, planteando protocolos en la praxis para una mejor comprensión en los instrumentos de valoración. Para concluir, la presencia del Trabajador Social en el proceso de la valoración gerontogeriátrica es fundamental en la comprensión de los resultados ya que, por la interferencia social en la aplicación de la normativa técnica, minimiza y agilita el protocolo para la consecución del informe del tamizaje social en los adultos mayores.

Palabras clave: trabajo social gerontológico, tamizaje social, plan de intervención individual, valoración gerontogeriátrica

\section{ABSTRACT}

The objective of the article was to determine the intervention processes of the interdisciplinary team in the gerontogeriatric evaluation of the residents of the Guillermina Loor de Moreno Gerontological Center. The work consists of two interrelated sections. The first describes the role of the Social Worker in the Gerontological Center (Gerontological Social Work) as leader of the interdisciplinary team. The second focuses on the gerontogeriatric evaluation obtained through the PIII (Comprehensive Individual Intervention Plan) carried out by the team's professionals, who act subject to the technical regulations of the MIES (Ministry of Economic and Social Inclusion). This allowed to better understand and process the intervention protocols in the field of the elderly. The descriptive methods - exploratory, bibliographic and inductive-deductive allowed to synthesize the information of each process, the interview technique facilitated acquiring relevant information in the care received by this vulnerable group, proposing protocols in praxis for a better understanding in the instruments of assessment. To conclude, the presence of the 
Social Worker in the gerontogeriatric assessment process is essential in understanding the results, since, due to social interference in the application of technical regulations, it minimizes and streamlines the protocol for obtaining the report of the social screening in older adults.

Keywords: gerontological social work, social screening, individual intervention plan, gerontogeriatric assessment

\section{Introducción}

El Trabajo Social es una praxis científica, con métodos de observación y comprobación, bien definidos e inherentes a un campo de acción concreto: el hombre integral, sus formas de comportamiento y sobre todo sus instituciones sociales y éticas, en cuanto deben servirle para la satisfacción de todas sus aspiraciones. Como profesión, se funda en el reconocimiento de la existencia de la dignidad humana y de su capacidad de superación. Sandoval (1970), menciona que "mediante procedimientos propios, ayuda a los individuos, grupos y comunidades a valerse por sí mismos y lograr su desarrollo integral, con especial énfasis en aquellos aspectos que más ayuda requieran".

El Trabajo Social es una disciplina científica que resulta de la práctica basada en la evidencia o viceversa, esto demuestra que se debe partir de una evidencia para obtener una práctica profesional o por lo tanto obtener la práctica a través de una evidencia científica. Esta profesión considera importante la observación de algún fenómeno o hecho, viendo al problema como una oportunidad para favorecer, desarrollar y fomentar cambios sociales que encamine a un bienestar para las personas.

\section{Funciones del Trabajador Social en los Centros Gerontológicos}

Las funciones del Trabajador Social en los Centros Gerontológicos varían desde la perspectiva institucional y profesional, entre ellas debe obtener una evaluación preliminar de los usuarios antes de armar su expediente, realizar informes sociales con las debidas recomendaciones, visitas domiciliarias en caso de tener familiares y procurar la participación y reinserción del adulto mayor en sus actividades diarias. Una de las labores más sobresalientes es coadyuvar en la readaptación y 


\section{0}

rehabilitación de los adultos mayores, acompañadas del equipo interdisciplinario para mejorar su condición de vida.

\section{Valoración Gerontogeriatrica}

Un elemento muy importante en la valoración gerontogeriátrica es la gerontología, que representa el estudio de los aspectos sociales, psicológicos y biológicos del envejecimiento. La gerontología analiza el proceso de envejecimiento en todas sus dimensiones: biológica, psíquica, económica, política, educativa y social (MIES, 2018). Es decir que estudia al adulto mayor en toda su potencialidad, enfocándose en sus áreas de estudios para priorizar sus necesidades en base a los resultados obtenidos.

Se trata del estudio de la vejez desde un enfoque interdisciplinario, siendo su propósito conocer el proceso de envejecimiento para la aplicación de test de valoraciones en la práctica profesional que permita mejorar la calidad de vida de los adultos mayores (LAFOREST, 1991). Bajo esta perspectiva surge la reflexión disciplinaria del Trabajo Social Gerontológico que Piña (2004), la define como "un campo de acción que apunta a potenciar el capital social individual, familiar, grupal y comunitario de los adultos mayores, mejorando la calidad de su vida social y su desarrollo humano, a través de una intervención social basada en enfoques epistemológicos, teóricos y metodológicos".

En la valoración Gerontogeriatrica es de suma importancia el trabajo en equipo, esto conlleva a la cadena de actividades que deben ir enfocadas en las valoraciones individuales de cada profesional, dentro de cada test de valoración existe una evaluación y por ende la supervisión; sin esto, no se podría realizar el tamizaje social, que es quien le da vida al informe de la valoración gerontogeriatrica.

\section{Tamizaje Social}

El tamizaje social también conocido como "tamizaje rápido" es la evaluación del adulto mayor en toda su magnitud o capacidad, es el resultado de todas las intervenciones, valoraciones, evaluación, planes y programas realizadas por los profesionales del equipo interdisciplinario. Zimmermman (2011), establece que "el tamizaje social en el adulto mayor es el proceso diagnóstico multidimensional y usualmente interdisciplinario, destinado a cuantificar en términos funcionales las 
capacidades y problemas médicos, mentales y sociales del adulto mayor con la intención de elaborar un plan de promoción, prevención, atención o rehabilitación, según corresponda".

En el tamizaje social se considera la atención integral que recibe el adulto mayor, entre otros factores que inciden en su desarrollo dentro del centro gerontológico para mejorar el proceso de envejecimiento.

\section{Atención Integral}

Es una intervención dirigida a personas adultas mayores, con un enfoque en el que se atienden todas las necesidades del paciente por completo, y no solo las necesidades médicas y físicas, sino a las que se ejecuta de manera permanente, incorporando actividades de promoción, prevención, recuperación, adaptación y rehabilitación en tres niveles: individual, familiar y comunitario (OPS, 1980).

La atención integral es un tipo de intervención orientada a los adultos mayores, proporcionando una gama de entornos que beneficien los procesos de intervención, enfocados en las necesidades de cada usuario, así mismo, el fortalecimiento y el apoyo en la capacidad técnica de los servicios que debe recibir el adulto mayor.

\section{Envejecimiento}

Proceso continuo en el que se desarrolla durante el curso de vida y que tolera cambios biológicos, fisiológicos, psicosociales y funcionales, las cuales se agrupan con interacciones dinámicas y permanentes entre el individuo y el medio en el que se desenvuelve. Desde una perspectiva biológica, el envejecimiento es la consecuencia de la acumulación de una gran variedad de daños moleculares y celulares a lo largo del tiempo, lo que lleva a un descenso gradual de las capacidades físicas y mentales, un aumento del riesgo de enfermedad, y finalmente a la muerte (OMS, 2018).

\section{Envejecimiento Saludable}

Es el proceso de fomentar y mantener la capacidad funcional que permite el bienestar en la vejez. Con este nuevo concepto, envejecer de manera saludable no significa envejecer sin enfermedades. Envejecer de manera saludable significa ser 


\section{2}

capaz de hacer durante el máximo tiempo posible las cosas a las que damos valor (OPS, 2019).

El envejecimiento saludable es una cadena por el cual el adulto mayor ha llegado a una etapa en donde ve como resultado todo el proceso de lo factores que incidieron en el transcurso de su vida, mostrando óptimos resultados en todos sus aspectos: nutricionales, físicos y cognitivos.

\section{Envejecimiento Activo}

Es el proceso por el que se optimizan las oportunidades de bienestar físico, social y mental durante toda la vida, con el objetivo de ampliar la esperanza de vida saludable, la productividad y la calidad de vida en la vejez. Esta definición no sólo contempla el envejecimiento desde la atención sanitaria, sino que incorpora todos los factores de las áreas social, económica y cultural que afectan al envejecimiento de las personas (OMS, 2010).

El envejecimiento activo es un medio fundamental para alcanzar el potencial significativo en el pleno gozo de la vida, enfocados en el estado de salud, físico, mental y de autonomía, orientado a la promoción y prevención de enfermedades para mejorar su calidad de vida.

\section{Método}

En la determinación de los procesos de intervención del equipo interdisciplinario para realizar la valoración gerontogeriatrica es muy importante resaltar el papel que cumple el Trabajador Social en la elaboración del tamizaje social. En el proceso se aplicaron métodos a nivel descriptivo, bibliográfico, inductivo-deductivo y exploratorio.

El método descriptivo consistió en evaluar cada intervención profesional de quienes realizan los test de valoración, así mismo la descripción de cada una de ellas en toda su dimensión de las características en particulares que dieron a conocer todo su desarrollo desde su naturalidad. Esto permitió conocer el proceso en las valoraciones individuales del equipo interdisciplinario en los 33 adultos mayores residenciales. Por otra parte, el trabajo se apoyó en investigaciones bibliográficas de fuentes anexadas en las rutas y protocolos de cada profesional en el campo de los adultos mayores, 
mostrando así aspectos necesarios en el accionar del equipo de trabajo para la consecución de los objetivos, partiendo de hechos particulares en la generación de conclusiones. También se aplicó como técnica la entrevista realizada al Trabajador Social, Psicólogo Clínico y el Médico de la institución.

\section{Resultados}

Es esencial conocer el proceso de intervención que debe adecuarse a la tipología del usuario en la valoración gerontogeriatrica; por ejemplo, los recursos que cuenta la institución y en el ámbito en el que se encuentre. Esta se define como un proceso diagnóstico multidimensional e interdisciplinario, diseñado para identificar y cuantificar los problemas físicos, funcionales, psíquicos y sociales que pueda presentar el anciano, con objeto de desarrollar un plan de tratamiento y seguimiento de dichos problemas, así como la óptima utilización de recursos con los cuales afrontarlos. Para que la valoración sea útil, se debe establecer un plan de seguimiento evolutivo que constate los beneficios de la aplicación de los determinados planes o tratamientos instaurados (DAVE, 2004).

La valoración gerontogeriátrica se deberá realizar cada tres meses y para su aplicación se consideran esferas de acción que intervienen en el estilo de vida de los adultos mayores:

\section{Figura 1}

Esferas de acción que intervienen en el estilo de vida de los adultos mayores

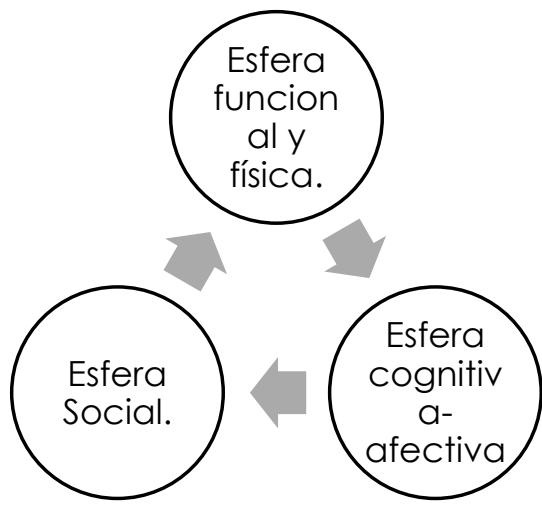




\section{4}

La esfera funcional y física, la valoración es un proceso direccionada a la recogida de información de la suficiencia que tiene el adulto mayor para desenvolverse y ejecutar sus actividades usuales dentro de su medio, de manera independiente. Éstas se distinguen entre las actividades básicas de la vida diaria y las actividades instrumentales de la vida diaria (Gonzalez, 2011).

Este proceso es dirigido por el profesional en terapista física y ocupacional, quienes cuentan con diferentes áreas para el desarrollo de actividades. En el área de fisioterapia el profesional realiza su planificación e intervención de seguimiento individual de las patologías de los adultos mayores para su rehabilitación, asimismo, la ejecución de programas individuales y atención terapéutica para el desarrollo de habilidades del adulto mayor. Es importante el acompañamiento técnico en la observación, abordaje e intervención de la evolución de los usuarios. Mientras que, en el área de terapia ocupacional y talleres, el profesional se encarga de evaluar y dirigir programas individuales adaptadas a cada necesidad del adulto mayor, valorizar las necesidades e intervenir de manera terapéutica para recuperar o mantener su autonomía.

La esfera cognitiva - afectiva en el área Psicológica es dirigida por el Psicólogo clínico, quien menciona que: "Siempre es necesario valorar a un adulto mayor para conocer en sí los factores funcionales, cognitivos y emocionales. En estas instancias en la esfera cognitiva se descarta si tiene una alteración cognitiva o deterioro cognitivo significativo, si se llegase a tener alguna alteración cognitiva hay que ver de dónde procede esa alteración. Una vez descartando estas alteraciones que tenga el adulto mayor se establece un diagnóstico para trabajar con una población, una vez obtenida la valoración se debe enfocar con la evolución, planificando actividades para su recuperación o el cumplimiento de los objetivos" (Mantuano, 2020).

En esta esfera el psicólogo debe elaborar un plan específico de su área y el plan acción en la intervención psicológica para el adulto mayor. Apoyar a la rehabilitación cognitiva y supervisar los talleres ocupacionales con la colaboración de la terapista ocupacional, dando seguimiento para el diseño y aplicación de estrategias de intervención para apoyar emocionalmente a los usuarios. 
El Trabajador Social dirige la esfera social, es importante el papel que cumple el profesional ya que dirige al equipo interdisciplinario en la valoración gerontogeriatrica y por ende el resultado del tamizaje social. El profesional de esta área coordina colectivamente la forma organizada y programada en las evaluaciones de las diversas actividades que se realizan en el centro residencial. Además de procurar la participación de trabajar con el equipo interdisciplinario, colaborando en la readaptación y rehabilitación de los adultos mayores por medio de la observación para luego abordar en una intervención.

El Trabajador Social también está encargado de realizar mapeos, visitas domiciliarias y coordinación de redes institucionales para brindar una atención al adulto mayor, gestionando interinstitucionalmente para mejorar la condición de vida de los usuarios del centro residencial. Aquí se realiza un estudio a nivel individual, familiar y comunal; lo cual permite identificar datos relevantes de la vida personal, sus vínculos familiares y las redes sociales con las que se cuenta.

Para realizar el proceso de intervención en la valoración gerontogeriatrica al ingreso del servicio, el centro gerontológico debe aplicar la ficha de aceptación, que consta de información relevante del adulto mayor, incluyendo las referencias familiares si tuviese, datos de identificación, entre otras. En el caso quien no cumpla con lo establecido para su ingreso, se debe orientar y derivar a instituciones pertinentes para el posible ingreso en la prestación de otros beneficios.

El centro de atención gerontológico debe verificar que los adultos mayores cuenten con la cédula de identidad o pasaporte como un derecho de ciudadanía. En caso de no contar con estos documentos, se debe coordinar con el registro civil o agilizar el proceso para su identificación en las instancias competentes.

Se debe elaborar el Plan de Intervención Individual Integral (PIII) por cada uno de los adultos mayores, donde se recogen las valoraciones, informaciones personales, objetivos, ideas, propuestas de intervención, apoyo personalizado y recomendaciones para que la persona adulta mayor adquiera el mayor grado de autogestión sobre su vida cotidiana para atender sus necesidades y colaborar en su proyecto de vida desde el impulso de la autonomía. Se realiza en base al criterio del equipo interdisciplinario, con la persona adulta mayor acompañado de su familia en caso de tenerla. 


\section{6}

Se debe realizar la planificación de actividades con una frecuencia mensual conforme al PIII para alcanzar las metas establecidas. La planificación debe contemplar los siguientes componentes:

- Salud

- Actividades de la vida diaria e instrumentales

- Cognitivas y afectivas

- Cuidados

- Derechos

- Espacios ambientales adecuados

- Educación

- Medios de vida

- Social

En este aspecto, se debe presentar los resultados de la valoración gerontogeriatrica a la persona adulta mayor y su familia en caso de tenerla, por medio de un informe semestral, con la finalidad de establecer una estrategia de trabajo para el cumplimiento de los objetivos a alcanzar a corto, mediano y largo plazo. La persona adulta mayor debe contar con un expediente único ordenado y en un espacio físico accesible a todo el equipo técnico.

En este contexto la ejecución del PIII integral del equipo interdisciplinario, ha contribuido en el desarrollo de la valoración gerontogeriatrica y el tamizaje social. Asimismo, el desempeño de los profesionales que conforman el Centro Gerontológico Guillermina Loor de Moreno: la Lic. Yvonne Bravo; Coordinadora y Trabajadora Social; la Lic. Nura Mero; Terapista Ocupacional, el Lic. Pablo Vélez; Terapista Físico, el Lic. Carlos Mantuano; Psicólogo Clínico y el especialista en medicina interna y magister en salud pública internacional el Dr. George Menéndez, conduce al trabajo en equipo para la consecución de los objetivos planteados, ampliando el resultado significativo de cada valoración para su evaluación. 


\section{Tabla 1}

Test de valoración gerontogeriatrica que se aplica en el Centro Gerontológico Guillermina Loor de Moreno

\begin{tabular}{|c|c|c|c|c|c|}
\hline \multicolumn{6}{|c|}{ USUARIO/A: APELLIDOS Y NOMBRES } \\
\hline EDAD: & & & $\begin{array}{l}\text { FECHA INICIO } \\
\text { DEL PLAN }\end{array}$ & & \\
\hline \multicolumn{6}{|c|}{ VALORACIÓN GERONTOGERIATRIA } \\
\hline $\begin{array}{l}\text { TAMIZAJE } \\
\text { SOCIAL }\end{array}$ & $\begin{array}{l}\text { ACTIVIDAD } \\
\text { INSTRUMENTAL } \\
\text { FUNCIONAL }\end{array}$ & CONGNITIVO & RECURSO SOCIAL & DEPRESIÓN & NUTRICIONAL \\
\hline \multicolumn{6}{|c|}{ ACTIVIDADES GENERALES ÁREA Y PERIODO } \\
\hline ÁREA & PRIMER TRIMESTRE & $\begin{array}{l}\text { SEGUNDO } \\
\text { TRIMESTRE }\end{array}$ & $\begin{array}{l}\text { TERCER } \\
\text { TRIMESTRE }\end{array}$ & $\begin{array}{l}\text { CUARTO } \\
\text { TRIMESTRE }\end{array}$ & ÁREA \\
\hline $\begin{array}{l}\text { Área } \\
\text { Salud y } \\
\text { Nutrición }\end{array}$ & & & & & \\
\hline $\begin{array}{l}\text { Área } \\
\text { Funcional }\end{array}$ & & & & & \\
\hline $\begin{array}{l}\text { Área } \\
\text { Cognitiva }\end{array}$ & & & & & \\
\hline
\end{tabular}

Nota. Centro Gerontológico Guillermina Loor de Moreno Portoviejo.

\section{Discusión}

A través de entrevistas a la Trabajadora Social de la Institución (Coordinadora del Centro Gerontológico Guillermina Loor de Moreno), se logró ampliar información sobre los procesos de intervención del equipo interdisciplinario y su función dentro de la misma.

Por consiguiente, en una de las entrevistas Bravo (2019) menciona:

"Es primordial que antes de entrar a la valoración gerontogeriatrica se debe enfocar en el Plan de Intervención Individual Integral, esta ficha es elaborada por el equipo interdisciplinario en donde cada profesional realiza su valoración por medio de herramientas como test de valoración que lo otorga el MIES (Ministerio de Inclusión Económica y Social) en la normativa técnica del manejo de los centros Gerontológicos residenciales". 


\section{8}

Una vez que estén aplicados todos los test de valoración, viene el tamizaje social, que es un diagnóstico que consta de una radiografía del sujeto, dándonos la pauta para identificar cómo está el adulto mayor para que cada profesional pueda elaborar un nuevo plan de trabajo para su aplicación y obtener una mejora o continuar con la evolución en el usuario.

Asimismo, Menéndez (2019), especialista en medicina interna y magister en salud pública internacional, explica:

"Es importante recalcar que los adultos mayores conforman un grupo de prueba poblacional y que la mayoría presentan enfermedades pluripatológicas dentro de su contexto. En lo que se valora a la condición subclínica puede comprometer la salud y la calidad de vida del adulto mayor, la valoración gerontogeriatrica constituye una herramienta diagnostica multi-funcional e interdisciplinaria cuya característica es evaluar al adulto mayor en toda su complejidad, tomando aspectos físicos mentales, socio familiares y funcionales, necesitando de un gran equipo de trabajo para su realización".

El adulto mayor es el objeto de estudio, considerando factores, influencias y patologías que se obtengan por la aplicación de test valorativos. Se puede realizar una mejor práctica en el desarrollo de los resultados obtenidos mediante la evaluación del usuario, el trabajo del equipo interdisciplinario debe ejercer una gran labor, cooperando y articulando sus actividades para mejorar la condición de vida de los adultos mayores.

La valoración gerontogeriatrica surge, además, como respuesta a la alta prevalencia en el anciano de necesidades y problemas no diagnosticados, mostrándose como un proceso diagnóstico dinámico y estructurado que permite detectar y cuantificar los problemas, necesidades y capacidades del anciano en las esferas clínica, funcional, mental y social para elaborar una estrategia interdisciplinar basada en ellos, de intervención, tratamiento y seguimiento a largo plazo con el fin de optimizar los recursos y de lograr el mayor grado de independencia $y$, en definitiva, calidad de vida. (Alarcón, 1993)

La valoración gerontogeriatrica resulta una herramienta de vital importancia con la finalidad de evaluar al usuario en el abordaje de sus problemas, cumpliendo criterios 
predominantes en base a un diagnóstico previo, para establecer un tratamiento adecuado y racional acorde a las necesidades del adulto mayor.

Es importante que el adulto mayor este informado de su condición y de cómo puede manejar, mantener o mejorar su estado de salud, considerando la información obtenida en el tamizaje social de las condiciones gerontogeriatricas, incluyendo orientaciones prácticas en el manejo del protocolo para disminuir el riesgo de su evaluación como un punto clave de un envejecimiento saludable (Goldberg, 1997). El accionar del profesional en Trabajo Social es fundamental en la difusión del resultado del tamizaje social, pues es quien deriva, informa, coordina y agilita los planes de intervención una vez obtenido el diagnóstico definitivo, que contribuye a la unidad de atención integral en los avances individuales para la mejora del adulto mayor.

\section{Conclusiones}

La intervención se centra en trabajar coordinadamente con el liderazgo del Trabajador Social para mover colectivamente al equipo interdisciplinario en la valoración gerontogeriatrica y la elaboración del tamizaje social. El análisis de esta combinación de factores muestra un escenario casi invisible en la labor que debe desarrollar el Trabajador Social en los centros gerontológicos residenciales, el proceso de intervención de cada profesional permite conocer la realidad de este sector.

Las valoraciones individuales del equipo interdisciplinario para la realización de la valoración gerontogeriatrica y el tamizaje social, permite mejorar el proceso de envejecimiento en los adultos mayores. Asimismo, el protocolo que debe seguir cada personal en una praxis profesional más centrada en los objetivos y funciones del Trabajador Social.

La presencia del Trabajador Social en el proceso de la valoración gerontogeriatrica es fundamental en la comprensión de los resultados establecidos por cada evaluación ya que, por la interferencia social en la aplicación de la normativa técnica, minimiza y agiliza el protocolo para la consecución del informe de tamizaje social en los adultos mayores. 


\section{Referencias}

Alarcón, S. (1993). Valoración Del Paciente Anciano. Barcelona: Masson.

Bravo, Y. (23 De Enero De 2019). Plan De Intervencion Individual Integral. (J. Giler, Entrevistador)

Dave, J. (2004). The Influence Gerontogeriatric. Valoracion Geriatrica Integral, 245.

Goldberg, T. (1997). Valoración Clínica Del Adulto Mayor. New York: Rochester.

Gonzalez, F. (201 1). Escalas De Valoracion Funcional. La Coruña: Galicia.

Inec, P. D. (2019). Instituto Nacional De Estadisticas Y Censos. Obtenido De Https://Www.Ecuadorencifras.Gob.Ec/Proyecciones-Poblacionales/

Laforest, J. (1991). Introducción A La Gerontología. Barcelona: Herder.

Mantuano, C. (7 de Febrero de 2020). Esfera Cognitiva- Afectiva. (J. Giler, Entrevistador)

Martinez, M. (2004). Trabajo-Social.Com. Obtenido De Https://Www.TrabajoSocial.Com/2011/10/Concepto-Del-Trabajo-Social.Html

Menéndez, G. (16 De Enero De 2019). Valoracion Gerontogeriatrica. (J. Giler, Entrevistador)

Mies. (2018). Normativa Técnica Para La Implementacion De Centros Residenciales. Quito : Editora Nacional .

OMS. (20 De Junio De 2010). Envejecimiento Activo En El Desarrollo Integral De Los Adultos Mayores. Obtenido De Https://Www.Hola.Com/Salud/EnciclopediaSalud/2010062045408/Mayores/Generales/Envejecimiento-Activo-Y-Saludable/

OMS. (2018). Convención Interamericana Sobre Los Derechos De Los Adultos Mayores. OPS. (1980). Organización Panamericana De Salud En Adultos Mayores. Norma Técnica. OPS. (2019). Organización Panamericana De La Salud. Obtenido De

Https://Www.Paho.Org/Hq/Index.Php?Option=Com_Content\&View=Article\&ld=1 3634: Healthy-Aging\&ltemid $=42449 \&$ Lang=Es

Piña, M. (2004). Trabajo Social Gerontológico. Buenos Aires: Espacio.

Sandoval, J. (1970). Asociación Nacional Mexicana De Trabajadores Sociales. Monterrey: Trujillo.

Zimmermman, J. (2011). Guía Médica De Atención Integral En El Adulto Mayor. Panamá: Donderis. 